\title{
Mechanism of Local Conformational Transitions in Poly(dialkylsiloxanes). Molecular Dynamics Simulations and Dynamic Rotational Isomeric State Approach
}

\author{
Ivet Bahar, ${ }^{\dagger}$ Neal Neuburger,,$^{\ddagger}$ and Wayne L. Mattice ${ }^{*, \ddagger}$ \\ Department of Chemical Engineering and Polymer Research Center, Bogazici University, \\ Bebek 80815, Istanbul, Turkey, and Institute of Polymer Science, University of Akron, \\ Akron, Ohio 44325-3909 \\ Received January 13, 1992; Revised Manuscript Received May 7, 1992
}

\begin{abstract}
Results from molecular dynamics simulations of isolated poly(dialkylsiloxane) chains are analyzed with emphasis on the conformational dynamics of a fragment of five repeat units, in which the alkyl group is tert-butyl. This fragment furnishes a typical example of a chain in which the bulky substituents restrict to a considerable extent the rotational motions of the backbone, which otherwise enjoys a high degree of flexibility. The rotational motions of the bonds flanking the oxygen atom are observed to be strongly correlated: the main mechanism of relaxation in the time range of picoseconds is the coupled counterrotations of those adjacent bonds. Examination of the molecular dynamics trajectories over much longer time scales, such as tenths of nanoseconds, reveals correlations between the rotational motions of neighboring repeat units, causing a relatively long-range coupling of backbone bonds along the chains, mainly between bonds $i$ and $i+3$. The orientational motion of the pair of bonds flanking the oxygen atom is analyzed within the framework of the dynamic rotational isomeric state formalism.
\end{abstract}

\section{Introduction}

In a recent work, molecular dynamics (MD) simulations were employed for a comparative study of the conformational statistics and unperturbed dimensions of a series of poly(dialkylsiloxane) (PDAS) chains, of the general formula $\mathrm{CH}_{3} \mathrm{O}\left[\mathrm{SiR}_{2} \mathrm{O}\right]_{x} \mathrm{CH}_{3}$, where $x$ is the number of repeat units and $\mathrm{R} \equiv \mathrm{C}_{m} \mathrm{H}_{2 m+1} \cdot{ }^{1}$ In the present work, the conformational dynamics of the same class of chains, with methyl, ethyl, propyl, butyl, isopropyl, isobutyl, and tertbutyl side groups, is considered, with special emphasis on poly(di-tert-butylsiloxane) (PDTBS), the member of the family which is distinguished by the strong correlations between neighboring bond rotations.

One of the main aspects of PDAS conformational statistics is the strong inderdependence of the rotational states of the pair of bonds centered about the oxygen atom, which is evident in the time-averaged probabilities for different pairs of values of the dihedral angles at these bonds. No distinct correlation between the states of the bonds flanking the Si atom is apparent, in the timeaveraged probabilities for this pair of bonds. The interdependence of the dihedral angles $\phi_{i}$ and $\phi_{i+1}$ associated with the respective bonds of type $\mathrm{Si}-\mathrm{O}$ and $\mathrm{O}-\mathrm{Si}$ becomes increasingly pronounced with increasing size of the side chains symmetrically attached to the $\mathrm{Si}$ atoms. Among the series of disubstituted polysiloxanes investigated by MD simulations, PDTBS presents the example with the bulkiest side groups. The probability distribution surface $P\left(\phi_{i}, \phi_{i+1}\right)$ obtained as a function of the torsional angles $\phi_{i}$ and $\phi_{i+1}$, with a methoxy-terminated fragment of $x=5$ repeat units, exhibits well-defined sequences of ridges and valleys, as a consequence of the strong coupling of the two dihedral angles.

All members of the PDAS series have an intrinsically flexible backbone, as a consequence of the long $\mathrm{Si}-\mathrm{O}$ bond, the lower barrier height for the intrinsic torsional potential at this bond, the absence of substituents at the oxygen atom, the large mean value of the $\mathrm{Si}-\mathrm{O}-\mathrm{Si}$ angle, and the unusually small force constant for bending at $\mathrm{Si}-\mathrm{O}-\mathrm{Si}$.

\footnotetext{
+ Bogazici University

$\ddagger$ University of Akron.
}

For this reason, there are only weak correlations in the rotations about the $\mathrm{Si}-\mathrm{O}$ bonds in poly(dimethylsiloxane) (PDMS). An increase in the bulk of the side chains enforces correlations due to the interaction of the side chains bonded to atoms $i$ and $i+2$. This physical origin of the correlations is different from that seen in polyethylene, where the backbone itself has strong conformational preferences, and the "side chains" are merely hydrogen atoms. Hence, we should not be surprised to find that the dynamic behavior of the PDAS series is much different from that of polymers with a backbone of carbon atoms. A more detailed analysis of the origin of the correlations of $\phi_{i}$ and $\phi_{i+1}$ in the PDAS with tert-butyl groups can be found in ref 1 .

The fact that the ridges in the probability surface are extended parallel to the line $\phi_{i}=-\phi_{i+1}$ implies that the rotational motion of bond $i$ is most likely accompanied by a counterrotation of bond $i+1$ or vice versa. That local conformational relaxation occurs via cooperative motion of near-neighbor bonds along the main chain is asserted in several studies. ${ }^{2}$ The coupling is generally manifested by two or more successive rotational transitions undergone within very short time intervals compared to the mean relaxation time of short segments. The coupling is mandatory in dense systems or in restricted geometry, as previous molecular dynamics simulations have demonstrated, ${ }^{3}$ but may become of secondary importance in the case of isolated chains, depending on the strength of the intramolecular constraints imposed by short-range energetics and chain connectivity. In this respect, polybutadiene is an interesting example, inasmuch as its conformational dynamics in restricted environment evolves through coupled counterrotations of bonds $i$ and $i+2$, although the single chain in the free state is subject to independent rotations of backbone bonds. ${ }^{4,5}$

The interdependence of adjacent bonds $i$ and $i+1$ is inherently present in most macromolecular systems. It is the basis of the Ising model or Markov chain model employed in chain statistics ${ }^{6}$ and stochastics. ${ }^{7}$ This nearest-neighbor interdependence is the fundamental postulate adopted in the dynamic rotational isomeric state formalism which has been developed ${ }^{8,9}$ for the treatment 
of local chain dynamics. Correlations between the rotational motion of bonds $i$ and $i+2$ on the one hand were first pointed out by Helfand, ${ }^{10}$ for $n$-alkanes, particularly when the central bond $i+1$ is in the trans state. Recent MD simulations confirm ${ }^{11}$ their existence but also indicate that they are operative over time ranges on the order of picoseconds and are not discernible throughout longer time windows. ${ }^{12}$ As to the cross-correlations between neighbors separated by a larger number of bonds, they are relatively difficult to detect, as they weaken with increasing separation. Three- or four-bond motions ${ }^{13}$ or crankshaftlike motions ${ }^{14}$ have also been proposed as conformational relaxation mechanisms favoring the localization of the motion along the chain, but they are not likely to occur in view of their high activation energies incompatible with experiments.

A rigorous method for the investigation of the type and mechanism of specific conformational relaxation processes in different polymers is the study of the MD trajectories of representative fragments. The PDAS series, and in particular PDTBS, which will be considered here present a challenging example in which a highly flexible backbone bearing bulky and highly articulated side groups is examined. The unusually high torsional flexibility of the main chain, supplemented by the very soft bending potentials at the $\mathrm{O}$ atom, will enhance the rate of rotameric transitions which, on the other hand, will be substantially hindered or restricted by the steric and energetic interferences of the side groups. Those structural and energetic factors combine to lead to a particularly interesting relaxational mechanism, as suggested by the regular and distinct shape of the probability distribution surfaces, from MD simulations. ${ }^{1}$. It is the purpose of the present work to exploit further the MD trajectories, in order to gain an understanding of the origins and implications of the observed behavior.

The present paper is organized as follows: Results from MD simulations are presented next, from the standpoint of the time evolution of rotameric states. In the same section, the spatial arrangement of the segments of the chains, which are surrounding the mobile repeat unit, will be analyzed, by following the displacement and reorientation of the ends of the representative fragment during rotameric transitions. A theoretical model accounting for the specific structure of the rotational space accessible to pairs of bonds flanking the $\mathrm{O}$ atom will be presented in the third section. In conformity with the equilibrium statistics treatment of PDAS chains, ${ }^{1}$ the conformational space spanned by two consecutive dihedral angles will be divided into 81 regions of equal size, with $40^{\circ}$ intervals along each direction. The stochastics of transitions between those regions of the conformational space will be considered within the framework of the dynamic rotational isomeric formalism.

\section{MD Simulations. Results and Discussion}

(a) General Picture. Two relaxational processes, those involving motions along a given ridge and those associated with the passages from one ridge to another, will be separately considered. Inasmuch as the motion along a given ridge necessitates negligibly small activation energies, that motion is expected to occur a few orders of magnitude faster than transitions between ridges. Time limitations of MD simulations allow for a systematic study of the characteristics of the motions over the ridges, while those between the ridges are accessible provided that simulations are performed for substantially long periods. The details of the MD simulations and their durations are given in earlier work.

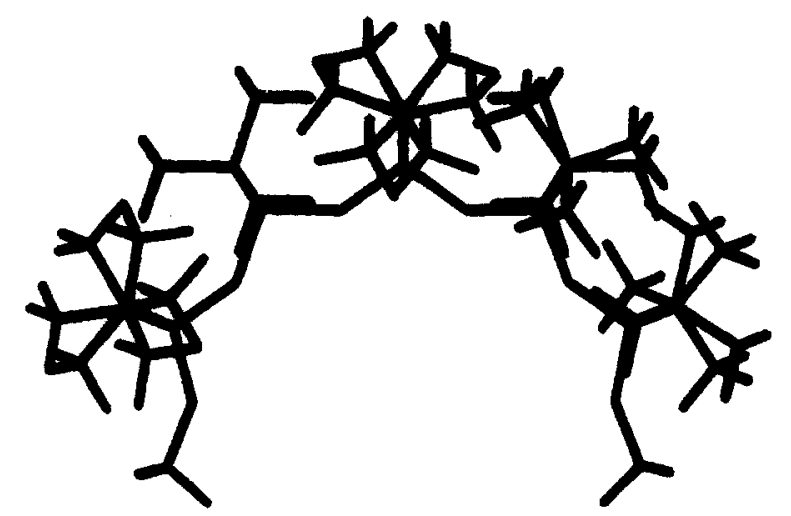

Figure 1. Schematic representation of a methoxy-terminated PDTBS fragment of five repeat units when all internal $\mathrm{Si}-0$ bonds have the trans conformation.

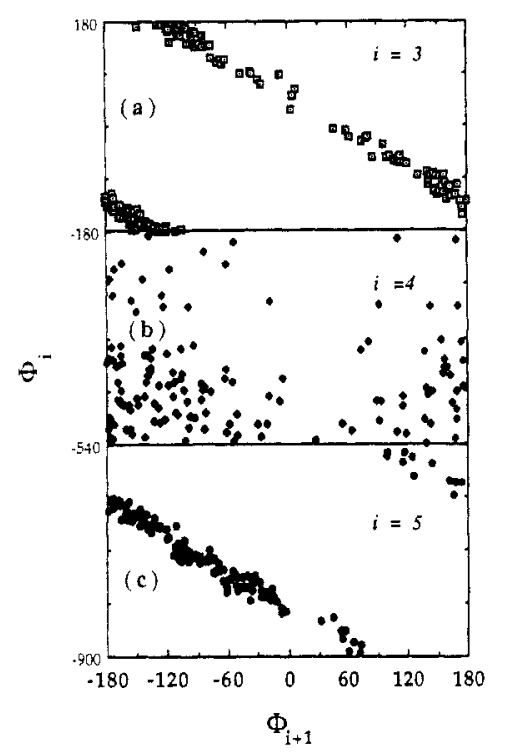

Figure 2. Snapshots of the joint rotational states $\left(\phi_{i}, \phi_{i+1}\right)$ of adjacent bonds in the PDTBS fragment, recorded with 100 -fs intervals, during a 20-ps portion of the MD trajectory for (a) $i$ $=3$, (b) $i=4$, and (c) $i=5$.

Figure 1 displays the planar trans form of the methoxyterminated PDTBS fragment of five repeat units, whose MD trajectory at $400 \mathrm{~K}$ has been considered. This conformation is highly improbable due to the overlap of the van der Waals volumes of the side-group atoms. The view in which the side groups eclipse each other is shown in the figure, in order to distinguish clearly the backbone. Backbone bonds are indexed from 1 to 10 , and $\phi_{i}$ is the torsional angle relative to the trans state, corresponding to bond $i$. Figure 2 illustrates the locations of the conformational space visited by the internal backbone bonds of this fragment, during an arbitrarily chose short portion (20 ps) of a MD trajectory. Parts a-c in Figure 2 represent the joint states assumed by the pair of bonds $(3,4),(4,5)$, and $(5,6)$, respectively. The points are snapshots recorded with time intervals of $100 \mathrm{fs}$. It is clearly apparent that the rotational states of the pair of bonds centered about the oxygen atom are strongly correlated, whereas the intermediate pair of bonds centered on a silicon atom almost randomly samples any region of conformational space. Thus, even a short simulation period of $20 \mathrm{ps}$ is able to reveal the major characteristics of the probability surfaces previously obtained with simulations of duration of tenths of nanoseconds: the occurrence of distinct highprobability ridges separated by well-defined channels in the case of ( $\mathrm{Si}-\mathrm{O}, \mathrm{O}-\mathrm{Si}$ ) bond pairs and a rather diffuse distribution of rotameric states for the pair $(\mathrm{O}-\mathrm{Si}, \mathrm{Si}-\mathrm{O})$. 


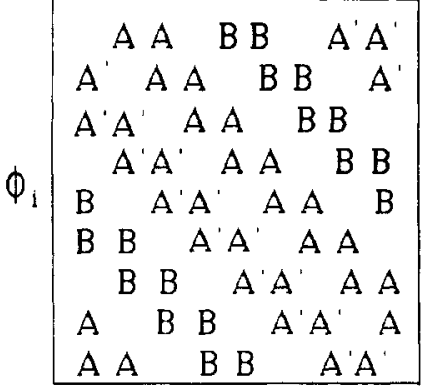

$$
\begin{aligned}
& O_{i+1}
\end{aligned}
$$

Figure 3. Schematic representation of the three types of ridges (macrostates) A, B, and $\mathrm{A}^{\prime}$ accessible to the pair of bonds (Si-O, $\mathrm{O}-\mathrm{Si}$ ). Each macrostate is composed of 18 microstates, covering regions of conformational space of size $\left(\Delta \phi_{i}, \Delta \phi_{i+1}\right)=\left(40^{\circ}, 40^{\circ}\right)$.

All of the points shown in part a (or part c) of Figure 2 belong to the same ridge, in view of the periodicity of the dihedral angle rotations. Thus, the upper points are nothing more than a continuation of the lower points or vice versa.

A convenient diagram for the description of the types of ridges accessible to the pair of bonds flanking an oxygen atom is shown in Figure 3. Three types of ridges $A, A^{\prime}$, and $B$ are present in the diagram. This scheme was deduced from the MD trajectories, taking account of the symmetry and periodicity properties of the conformational space of consecutive bond rotations. Ridges $A$ and $A^{\prime}$ are mirror images. They are identical insofar as the heights, amplitudes, and structural details are concerned. However, it is preferable to assign them different symbols (here the prime sign) when considering the dynamics of the fragment, since they represent two sets of conformations separated by high-energy walls.

The gradual evolution from one ridge to another may be followed by examination of a given ( $\mathrm{Si}-\mathrm{O}, \mathrm{O}-\mathrm{Si}$ ) bond pair over a longer time interval. Figure 4 a shows an example of the passage for the pair of dihedral angles $\left(\phi_{3}\right.$, $\phi_{4}$ ) from ridge $A$ to ridge $B$ and then to ridge $A^{\prime}$, observed during the time interval $60 \mathrm{ps} \leq t \leq 80 \mathrm{ps}$, of the MD trajectory. The empty circles which are situated along ridge A represent the snapshots recorded between 60.0 and $66.4 \mathrm{ps}$; the filled circles on ridge $B$ stand for those between 68.2 and 80.0 ps. The passage from $A$ to $B$ is effectuated during the short time interval $66.4 \mathrm{ps} \leq t \leq$ $68.2 \mathrm{ps}$, as indicated by the plus signs. This figure suggests that the pair of bonds ( $\mathrm{Si}-\mathrm{O}, \mathrm{O}-\mathrm{Si})$ spend a considerable time on a given ridge before moving to another ridge. The analysis of the MD trajectory of the same pair of bonds over a wider time range, say $20 \mathrm{ps} \leq t \leq 132 \mathrm{ps}$, confirms that the residence time over a given ridge is substantially large, as illustrated in Figure $4 \mathrm{~b}$. In this figure, the symbols $\Delta, X, \Theta$, and $O$ correspond to the respective time intervals $(20.0,66.4),(66.6,68.2),(68.4,99.6)$, and $(99.8,132.2)$, in picoseconds. Now we turn our attention to the highfrequency fluctuations of dihedral angles, which take place during residence on a given ridge.

(b) Motion along the Ridges. For a better understanding of the mechanism and time scale of motions occurring along a given ridge, we analyze the successive changes in the dihedral angles of a given pair of bonds centered about the $\mathrm{O}$ atom, over short time ranges of a few picoseconds.

Bonds $(3,4)$ in Figure 1 and the portion of the trajectory between 15 and 20 ps are taken for that purpose, inasmuch as the analysis in the preceding section has shown that the pair of dihedral angles $\left(\phi_{3}, \phi_{4}\right)$ is confined to move in a fixed ridge during this period. Figure $5 a$ displays the time
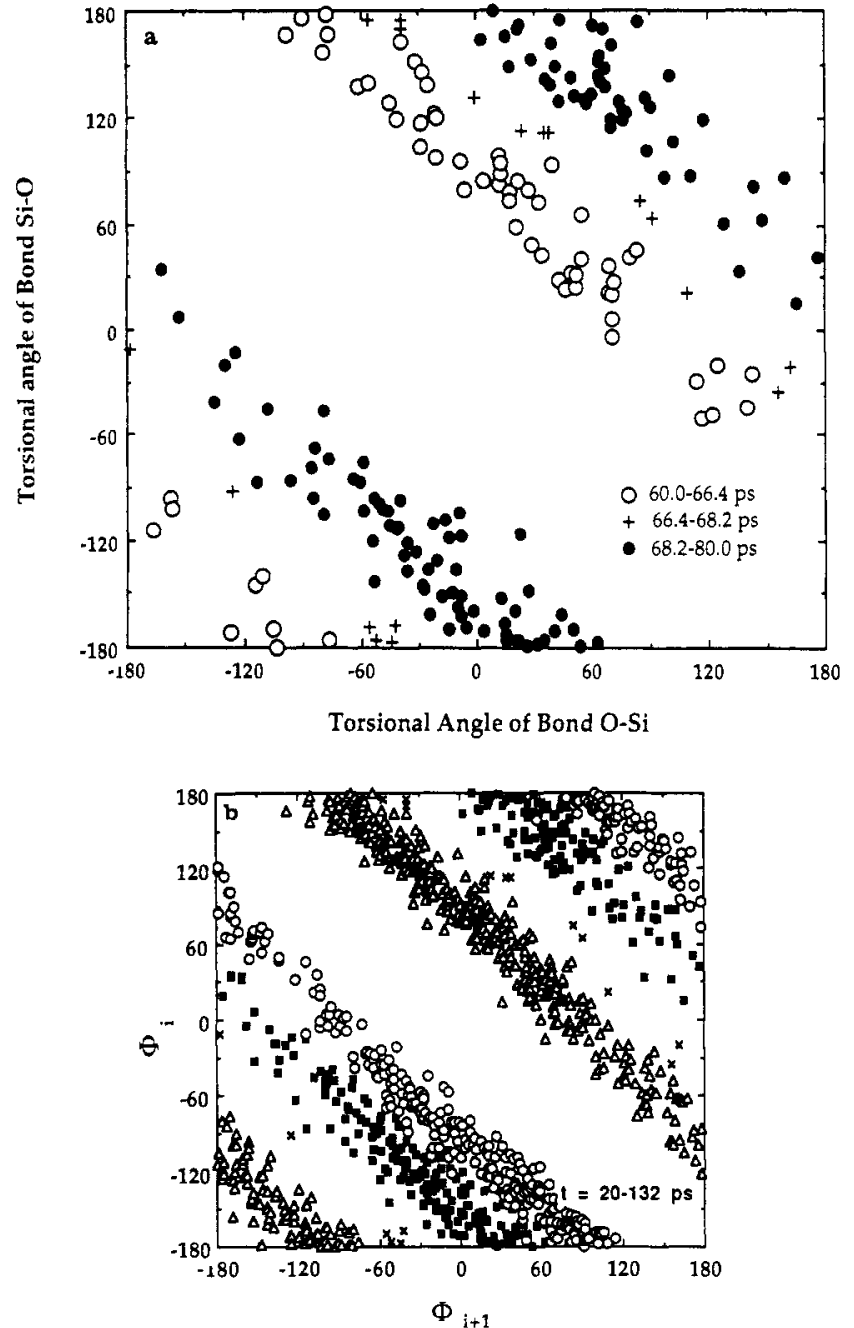

Figure 4. (a) Time evolution of joint states $\left(\phi_{3}, \phi_{4}\right)$ of the bond pair $(3,4)$ in the PDTBS fragment, observed during the time range $60 \mathrm{ps} \leq t \leq 80 \mathrm{ps}$ of the MD trajectory. (b) Same as Figure $4 \mathrm{a}$ but for the time interval $20 \mathrm{ps} \leq t \leq 132 \mathrm{ps}$.

dependence of the coupled torsional motion of the two bonds. Empty and filled circles represent the two different dihedral angles, recorded every $100 \mathrm{fs}$. The strong correlation between the torsional motion of the adjacent bonds is clearly apparent. A positive rotation undergone by one bond is generally accompanied by a negative rotation by the other bond. Two complete revolutions are chosen as the ordinate scale in the figure, instead of the usual range $\left(-180^{\circ},+180^{\circ}\right)$, in order to give an adequate description of the fluctuations occurring near the values of $\pm 180^{\circ}$, which are frequently assumed by both dihedral angles. The equivalent figure for the pair of bonds $(5,6)$, during the same time interval, is shown in Figure 5b. An interesting feature observed in these figures is the large amplitude of oscillations, of about $100^{\circ}$ rotations, which occur in spite of the high frequency of those motions. Full rotations of $360^{\circ}$ are achieved somewhat faster for bonds near the ends of the chain.

Rapid oscillatory motions of large amplitude along a given ridge may be rationalized by considering the spatial arrangement of the chain segments surrounding a given ( $\mathrm{Si}-\mathrm{O}, \mathrm{O}-\mathrm{Si}$ ) bond pair, following the coupled rotation of the central bonds. For a clearer visualization, we concentrate on the motion of the central bond pair in a PDMS fragment of six repeat units. All bonds other than those in the central ( $\mathrm{Si}-\mathrm{O}, \mathrm{O}-\mathrm{Si}$ ) bond pair are held in the trans position. The central bonds are rotated in conformity with their values along a given ridge. Three example 

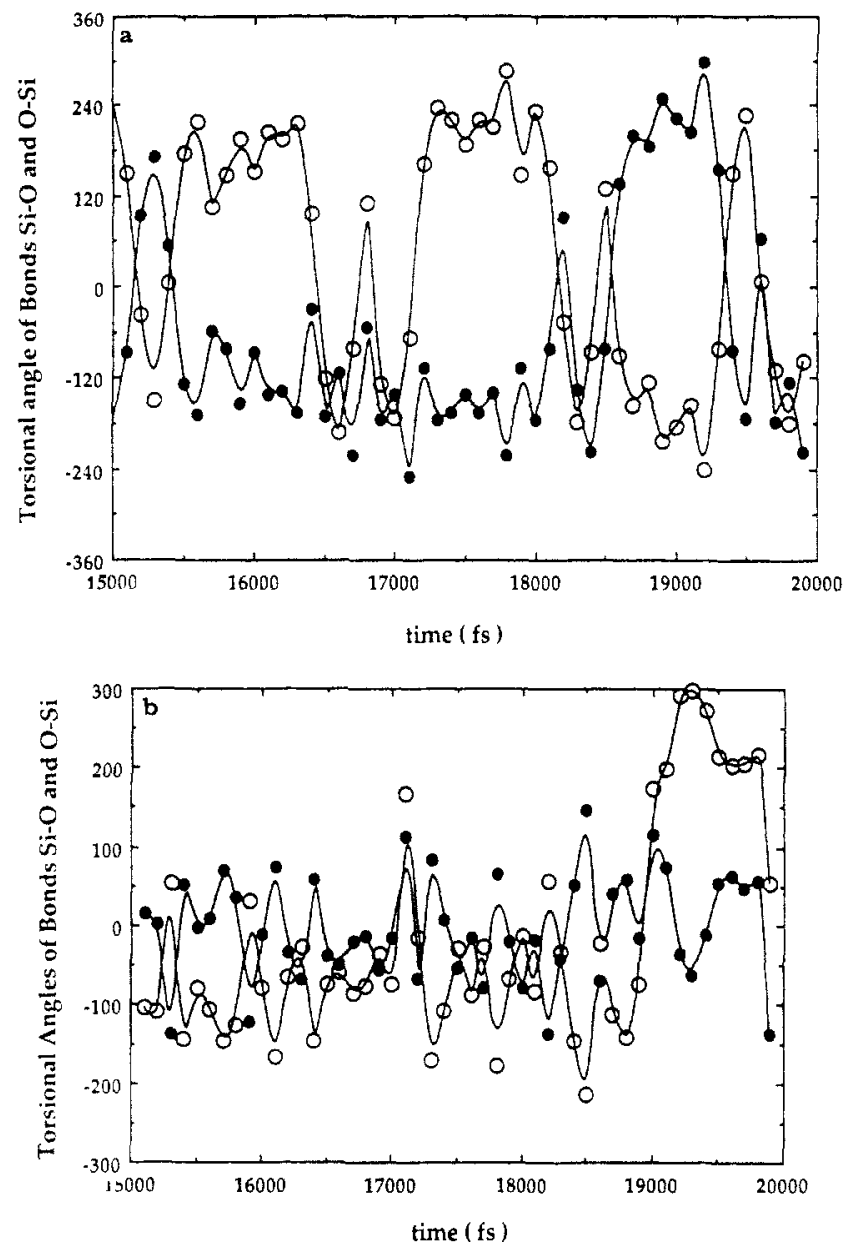

Figure 5. (a) Time dependence of the dihedral angles at bonds 3 (empty circles) and 4 (filled circles) in PDTBS, indicating strong correlation between the torsional motion of bonds $\mathrm{Si}-\mathrm{O}$ and $\mathrm{O}$ $\mathrm{Si}$. (b) Same as Figure $5 \mathrm{a}$ but for the pair of bonds $(5,6)$.

successive conformers with the respective torsional angles $\left(0^{\circ}, 176^{\circ}\right),\left(51^{\circ}, 139^{\circ}\right)$, and $\left(100^{\circ}, 100^{\circ}\right)$ are shown in Figure $6 a$. Those three values belong to ridge $B$ and are thus representative of a typical motion along a given ridge. The left-hand portions of the segments are superposed to obtain a clearer picture of the net displacement and reorientation of the right half, as the two central bonds sample different pairs of torsional angles, along the given ridge. Figure $6 \mathrm{~b}$ displays the top view of the same set of conformers. An interesting feature observed in these figures is the relatively small displacement of the bonds surrounding the central rotating bonds. Thus, the coupled counterrotation of the pair of bonds centered about the oxygen atom leads to short excursions of the near neighbors that are expected to be easily accommodated by the connected tails in a long chain. It should be noted that the torsional motion of the central bonds is reflected not only on the left-hand side portion of the segment, as is artifically shown in parts a and b of Figure 6, but also on both directions. The more or less equal distribution of the conformational change over both sides facilitates even further the readjustment of the tails. The unusually large value of the $\mathrm{Si}-\mathrm{O}-\mathrm{Si}$ bond angle contributes to the small motion of the tails.

(c) Motion between Ridges. The passages between ridges are several orders of magnitude slower than the oscillatory motions along a given ridge. In order to keep track of the time dependence of the motion between ridges, we assign a new variable $\Psi$ to a given pair of dihedral
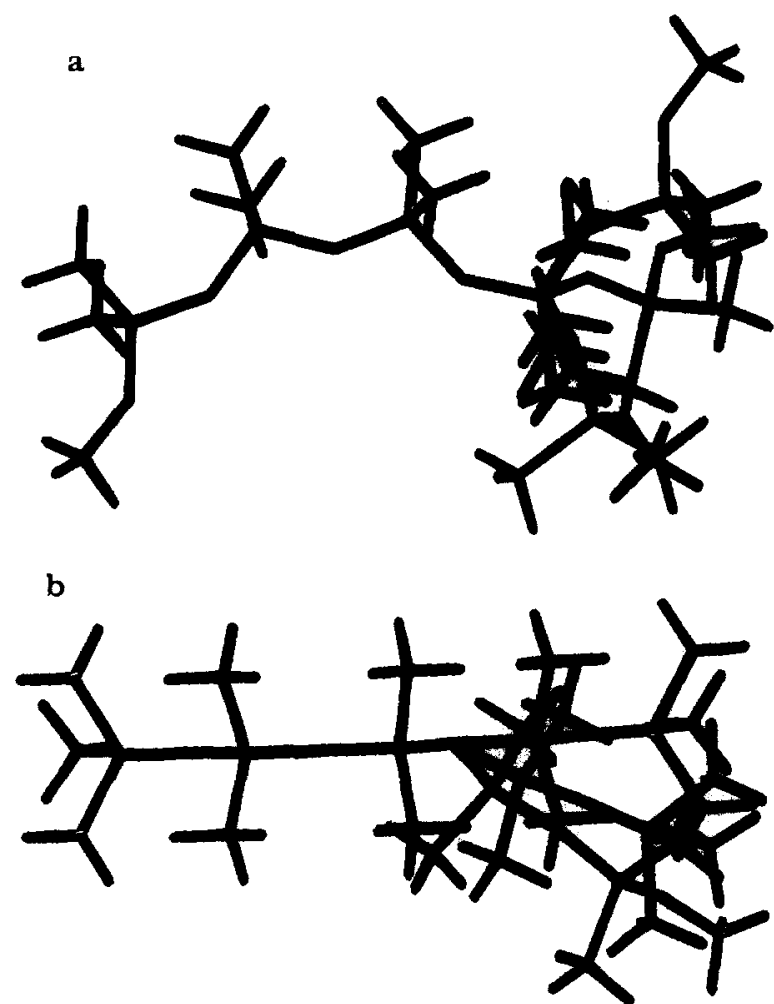

Figure 6. (a) Schematic representation of three example successive conformers with the respective torsional angles $\left(0^{\circ}\right.$, $\left.176^{\circ}\right),\left(51^{\circ}, 139^{\circ}\right)$, and $\left(100^{\circ}, 100^{\circ}\right)$, belonging to ridge $B$ and thus representative of a typical motion along a given ridge. The left-hand portions of the segments are superposed. (b) Top view of the same set of conformers as displayed in Figure 6a.

angles $\left(\phi_{i}, \phi_{i+1}\right)$ centered about 0 , such that

$$
\Psi= \begin{cases}\phi_{i}+\phi_{i+1}-180^{\circ}, & \phi_{i}+\phi_{i+1} \geq 0 \\ \phi_{i}+\phi_{i+1}+180^{\circ}, & \phi_{i}+\phi_{i+1}<0\end{cases}
$$

The above operation yields the projection of the given pair of bonds $\left(\phi_{i}, \phi_{i+1}\right)$ shown in Figure $4 \mathrm{a}$,b, along the direction of the diagonal $\phi_{i}=-\phi_{i+1}$, on the right ordinate. Hence, any point in the upper left triangle of Figure 4a,b is moved to the right, along a line of slope -1 , until it intersects the right boundary of the figure. The value of $\Psi$ for the point is the value of $\phi_{i}$ at this intersection with the right boundary. Any point in the lower left triangle is moved to the left, along a line of slope 1 , until it intersects the left boundary of the figure, and the value of $\phi_{i}$ at this interaction becomes the value of $\Psi$. Accordingly, the respective ridges $\mathrm{A}, \mathrm{B}$, and $\mathrm{A}^{\prime}$ are characterized by the intervals $-140^{\circ} \leq \Psi \leq-60^{\circ},-20^{\circ} \leq \Psi \leq 60^{\circ}$, and $100^{\circ} \leq$ $\Psi \leq 180^{\circ}$, approximately. ${ }^{15}$

Figure 7 displays the time evolution of $\Psi$ observed during the time interval $20 \mathrm{ps} \leq t \leq 280 \mathrm{ps}$ of the complete MD trajectory, for two consecutive pairs of bonds centered about oxygen. The circles represent snapshots recorded at intervals of $300 \mathrm{fs}$. The black and gray circles correspond to the pair of bonds $(3,4)$ and $(5,6)$, respectively, of the fragment shown in Figure 1. The following two important pieces of information may be extracted from this type of figure:

(i) From the examination of a given set of points, either black or gray, over longer time ranges, say the whole period of the MD simulations, and then taking averages over both pairs centered about $O$, it is possible to estimate the mean residence time on a ridge. In the present case, both pairs of bonds exhibit about eight transitions during $0.26 \mathrm{~ns}$, which leads to a mean residence time of approximately $0.03 \mathrm{~ns}$ in a given ridge, for PDTBS. Here, not the exact 


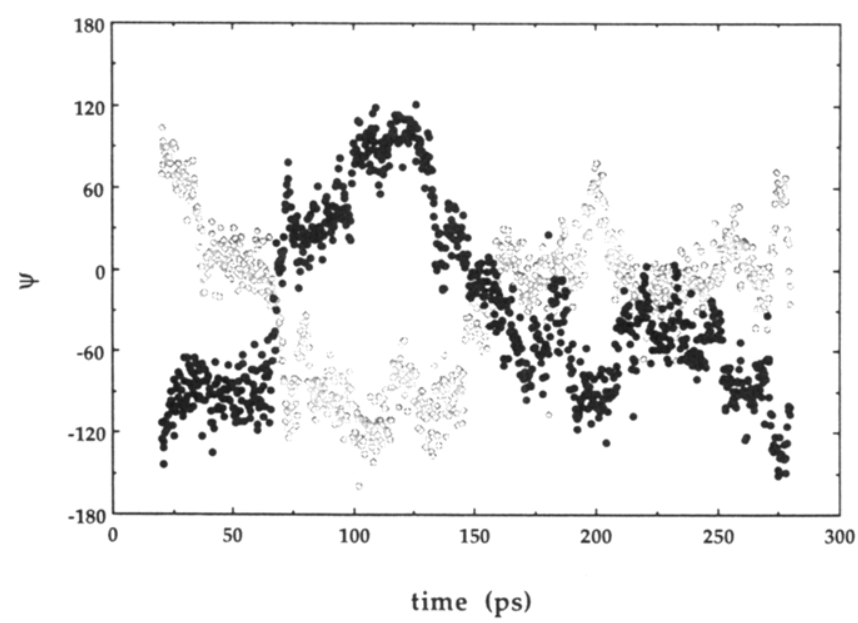

Figure 7. Time dependence of $\Psi$ observed during $20 \mathrm{ps} \leq t \leq$ 280 ps. $\Psi$ is the variable obtained for a given joint state $\left(\phi_{i}, \phi_{i+1}\right)$, from the projection of the corresponding point shown in Figure $4 \mathrm{a}, \mathrm{b}$, on the right ordinate, along the direction of the diagonal $\phi_{i}=-\phi_{i+1}$. Accordingly, the respective ridges $\mathrm{A}, \mathrm{B}$, and $\mathrm{A}^{\prime}$ are characterized by the intervals $-140^{\circ} \leq \Psi \leq-60^{\circ},-20^{\circ} \leq \Psi \leq 60^{\circ}$, and $100^{\circ} \leq \Psi \leq 180^{\circ}$, approximately. The circles represent snapshots recorded at intervals of $300 \mathrm{fs}$. The black and gray circles correspond to the pair of bonds $(3,4)$ and $(5,6)$, respectively, of the fragment shown in Figure 1.

absolute value of the residence time but its order of magnitude, relative to the fast motions of equal amplitude but restricted in a given ridge, is of interest: The time scale of transitions between ridges is about 3 orders of magnitude larger.

(ii) Simultaneous inspection of both sets of points in Figure 7 reveals the strong coupling between the adjacent pairs of bonds. In fact, it is clearly seen from Figure 7 that passages of the pair of bonds $(3,4)$ from one ridge to another are systematically accompanied by the transitions of the pair of bonds $(5,6)$. This strong interdependence of the motions of the adjacent repeat units brings into consideration a new type of coupling between backbone bonds, which is of longer range and lower frequency, compared to those encountered in previous studies: A coupling between bonds $i$ and $i+3$ takes place, through the strong orientational correlations of adjacent repeat units.

\section{Dynamic Rotational Isomeric State Treatment}

(a) General Formulation. The dynamics of conformational transitions undergone by the pair of bonds centered about $\mathrm{O}$ is approximated by a simple model based on a discrete number of passages between different regions of conformational space. The space is defined by the rotational angles of the two bonds and divided into 81 regions of equal size, in agreement with the recent rotational isomeric state formulation proposed for the members of the PDAS series. ${ }^{1}$

Following the diagram given in Figure 3, a total of $N=$ 54 rotational isomeric states are defined for the bond pair, the remaining 27 regions of the conformational space being occupied by high-energy domains, which naturally may not be identified by stable states. The stable $54 \mathrm{mi}-$ crostates are grouped into three macrostates defined by the ridges $\mathrm{A}, \mathbf{A}^{\prime}$, and $\mathrm{B}$. Equal equilibrium probabilities are conveniently assigned to each of the microstates belonging to a given macrostate. High-energy barriers separate the macrostates, while passages between microstates on a given ridge require very low activation energies, in conformity with the results from MD simulations. Nevertheless, a model comprising 54 distinct states will be employed, inasmuch as the analytical treatment of the diffusion along a given ridge will be undertaken below, which requires that we distinguish between the various regions of space within a given ridge.

The stochastic process of conformational transitions of the pair of bonds ( $\mathrm{Si}-\mathrm{O}, \mathrm{O}-\mathrm{Si}$ ) is described by the master equation

$$
\mathrm{d} \mathbf{P}(t) / \mathrm{d} t=\mathbf{A} \mathbf{P}(t)
$$

where the vector $\mathbf{P}(t)$ represents the array of the instantaneous probabilities of the $N$ states and the $N \times N$ transition rate matrix $\mathbf{A}$ is composed of the rate constants associated with various transitions. Let the indices $\alpha, \beta$, ..., $N$ indicate the isomeric states accessible to the pair of bonds (Si-O, O-Si). The off-diagonal $\alpha \beta$ th element of $\mathbf{A}$, $A_{\alpha \beta}$, is the rate constant for the transition from state $\beta$ to state $\alpha$ and the diagonal elements $A_{\alpha \alpha}$ are given by $A_{\alpha \alpha}=$ $-\sum A_{\alpha \beta}$, where the summation is performed over all $\beta \neq \alpha$. In agreement with previous studies, only single-bond rotations are assumed at a time and the elements of $\mathbf{A}$ corresponding to simultaneous transitions of both dihedral angles are equated to zero. This assumption does not exclude the possibility of coupled rotations of the two adjacent bonds, with an infinitesimally small time interval, as in the present case. In general, four possible paths for conformational change are accessible to each state, which result from $\pm 40^{\circ}$ (or $\pm 80^{\circ}$ ) rotational jumps of either bond in the pair; motions along the ridges occur through successions of steps along the vertical and the horizontal directions of the diagram shown in Figure 3.

The formal solution to eq 2 is

$$
\mathbf{P}(t)=\exp (\mathbf{A} t) \mathbf{P}(0)=\mathbf{B} \exp (\Lambda t) \mathbf{B}^{-1} \mathbf{P}(0)
$$

where $\mathbf{B}$ and $\Lambda$ are obtained from the diagonalization of A as the matrix of the eigenvectors and the diagonal matrix of the eigenvalues, respectively, and $\mathbf{B}^{-1}$ is the inverse of B. The product $\mathbf{B} \exp (\Lambda t) \mathbf{B}^{-1}$ is nothing more than the transition or conditional probability matrix $\mathbf{C}(t)$. The element $C_{\alpha \beta}(t)$ or $C(\alpha, t / \beta, 0)$ of $\mathbf{C}(t)$ denotes the conditional probability of occurrence of state $\alpha$ at time $t$, given the original state $\beta$.

The orientational autocorrelation function $M(t)$ for a vector $\mathbf{m}$ rigidly embedded in the chain is readily evaluated as a function of time, from the weighted additive contribution of all types of conformational transitions, as

$$
\begin{gathered}
M(t) \equiv\langle\mathbf{m}(0) \cdot \mathbf{m}(t)\rangle=\sum_{\alpha} \sum_{\beta}\left(\mathbf{m}_{\alpha} \cdot \mathbf{m}_{\beta}\right) C_{\alpha \beta}(t) P_{\beta}(0) \\
M(t)=\sum_{\alpha} \sum_{\beta} \sum_{\gamma}\left(\mathbf{m}_{\alpha} \cdot \mathbf{m}_{\beta}\right) B_{\alpha \gamma} \exp \left\{\lambda_{\gamma} t\right\} B_{\gamma \beta}{ }^{-1} P_{\beta}(0) \\
M(t) \boxminus \sum_{\gamma} k_{\gamma} \exp \left\{\lambda_{\gamma} t\right\}
\end{gathered}
$$

Here $\lambda_{\gamma}$ is the $\gamma$ th element of $\Lambda$, which is equal to the negative of the frequency of the relaxational mode $\gamma, \mathbf{m}_{\gamma}$ is the vectorial representation of $\mathbf{m}$ in a fixed frame when the unit in which it is embedded is in state $\gamma$, and $k_{\gamma}$ is the amplitude factor associated with mode $\gamma$. As may be seen from the comparison of eqs 5 and $6, k_{\gamma}$ depends on $\mathbf{m}, \mathbf{B}$, and the equilibrium probabilities $P_{\beta}(0)$ which are the elements of $\mathbf{P}(0)$. For the particular case of the vector $\mathbf{m}$ coupled to a given pair of bonds ( $\mathrm{Si}-\mathrm{O}, \mathrm{O}-\mathrm{Si}$ ), the summations in eqs $4-6$ are performed over the 54 stable conformations of the repeat unit.

(b) Illustrative Calculations. When the rate of transitions between ridges (i.e., for transitions of the type 


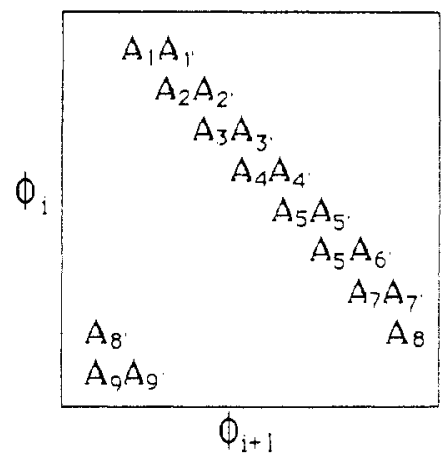

Figure 8. Schematic representation of the ridge $A$ in which the 18 different microstates are indexed as $1,1^{\prime}, 2,2^{\prime}, \ldots, 9^{\prime}$.

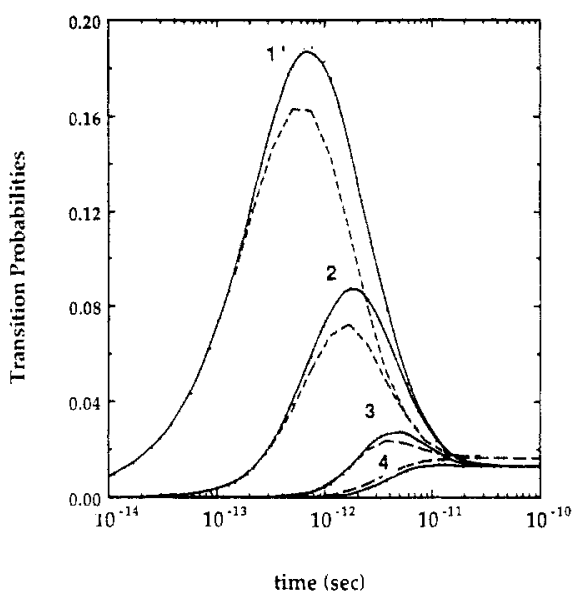

Figure 9. Time dependence of the transition probabilities $C(\alpha, t)$ $\beta, 0)$, with $\alpha=1^{\prime}, 2,3$, and 4 and $\beta=1$ for $300 \mathrm{~K}$. Solid and dashed curves are obtained with the two respective sets of energy parameters I and II, listed in Table I.

$A \rightleftharpoons B \rightleftharpoons A^{\prime}$ in Figure 3 ) is slow compared to the rate of motion along a particular ridge (i.e., visiting all of the 18 letters " $A$ " on ridge $A$ in Figure 3, given the occupation of ridge $A$ at zero time), the decay of the orientational autocorrelation function will be governed by the latter process. We now proceed with the analysis when motion along one of the ridges controls the dynamics.

In order to analyze the stochastic process of coupled rotations during residence on a given ridge, transition probabilities for the passage between different states along a given ridge were evaluated as a function of time. Figure 8 shows ridge $A$ in which the different microstates are indexed as $1,1^{\prime}, 2,2^{\prime}, \ldots, 9^{\prime}$. All microstates are assumed to have equal equilibrium probabilities and are separated by energy barriers of the same height. Mainly three distinct transition probabilities are considered, which correspond to passages from the original state 1 to the final states $1^{\prime}, 2,3$, and 4 . From symmetry and periodicity considerations, it is clear that transitions from state $i$ to either $(i+j)$ or $(i-j), 1 \leq j \leq 4$, follow the same stochastics, and for $j>4$, the shortest path which is in the opposite direction is preferred. In this respect, it is unnecessary to consider the passage from state 1 to states $5-9$, for example, since they are equivalent to passages to states $4-1$.

The time dependence of the transition probabilities $C(\alpha, t / \beta, 0)$, with $\alpha=1^{\prime}, 2,3$, and 4 and $\beta=1$, is displayed in Figure 9. Results are obtained for $300 \mathrm{~K}$, assuming all microstates belonging to a given ridge to possess identical equilibrium energies and to be separated by activation energies of $0.1 \mathrm{kcal} / \mathrm{mol}$. Solid and dashed curves are obtained with the two respective sets of energy parameters I and II, listed in Table I. Those values roughly approx-
Table I

Energy Parameters (kcal/mol)

\begin{tabular}{ccccccc}
\hline & \multicolumn{2}{c}{ equilibrium energies } & & \multicolumn{2}{c}{ barrier height } \\
\cline { 2 - 3 } ridge & \multicolumn{1}{c}{ I } & II & & passage & I & II \\
\hline $\mathrm{A}$ & 0.0 & 0.0 & & $\mathrm{~A} \leftrightarrow \mathrm{A}^{\prime}$ & 3.0 & 3.0 \\
$\mathrm{~A}^{\prime}$ & 0.0 & 0.0 & & $\mathrm{~A} \leftrightarrow \mathrm{B}$ & 1.0 & 0.5 \\
$\mathrm{~B}$ & -0.5 & -0.2 & $\mathrm{~B} \leftrightarrow \mathrm{A}^{\prime}$ & 1.0 & 0.5
\end{tabular}

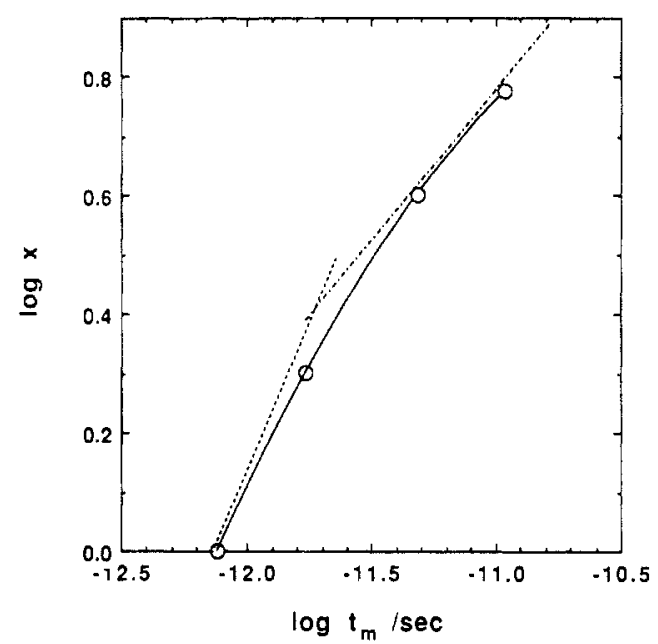

Figure 10. Dependence of $t_{\mathrm{m}}$ on the distance traveled along a ridge. The maximum of the curves in Figure 9 is denoted by $t_{\mathrm{m}}$, and $2 x$ characterizes a unit displacement of size $\left(\Delta \phi_{i}, \Delta \phi_{i+1}\right)=$ $\left(+40^{\circ},-40^{\circ}\right)$ along a ridge. The two dashed lines have slopes 1 and 0.5 .

imate the recent results from MD simulations obtained for PDtBS. ${ }^{1}$ The value of $10^{12} / \mathrm{s}$ is used for the front factor of the expressions for the rate constants in the transition rate matrix $\mathbf{A}$, while the activation energies therein are calculated from the tabulated barrier heights, relative to energy minima.

The transition probability curves obtained with either set of energy parameters exhibit the same qualitative features: A rise to a maximum value in $C(\alpha, t / \beta, 0)$ is observed at short times, followed by the decay to the equilibrium probability $P_{\alpha}(0)$ at long times. The convergence to the equilibrium value $P_{\alpha}(0)$ appears as a strictly increasing function only in the case of the transition between the most distant microstates 1 and $4 .{ }^{16}$ Otherwise, there is momentarily an enhanced probability of the state $\alpha$, which is attributed to the very low barrier height between states $\alpha$ and $\beta$. Transition probability curves of similar shape were calculated for the escape from the $\mathrm{g}^{+} \mathrm{g}^{-}$state in polyethylene bonds, which again necessitates very small activation energies. ${ }^{9}$

The characteristic times $t_{\mathrm{m}}$ corresponding to the maxime of the transition probability curves obtained for different amplitude motions give an indication of the stochastic nature of the diffusion process along a given ridge. The microstates $1,2, \ldots, 9$ in Figure 8 are separated by angular displacements $\left(\Delta \phi_{i}, \Delta \phi_{i+1}\right)$ of $\left(40^{\circ}, 40^{\circ}\right)$. Accordingly, it is possible to identify each step between consecutive microstates, by a unit displacement in the rotational subspace of a given ridge. The transitions between microstates differing by the prime sign may be viewed as half steps. Thus, the transitions studied in Figure 9, correspond to displacements of $x / 2$ steps where $x=1,2,4$, and 6 . The characteristic times $t_{\mathrm{m}}$ obtained with set $\mathrm{I}$ in Table I for different $x$ values are displayed in Figure 10, in the form of $a \log x$ vs $\log t_{\mathrm{m}}$ plot. The slope of the curve in the figure decreases approximately from 1 to 0.5 as indicated by the two dashed lines. It is interesting to note that the value of 0.5 , which is approached during transitions 


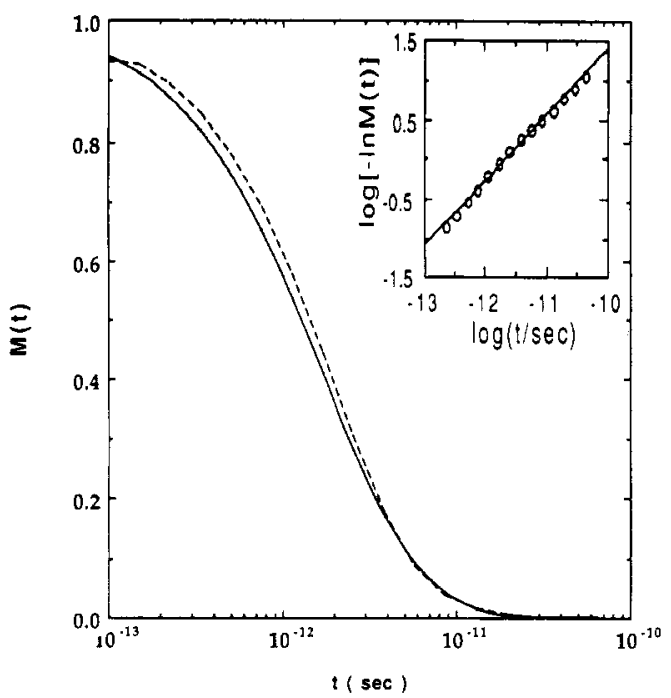

Figure 11. Time decay of the orientational autocorrelation function $M(t)$ for the bond vector $\mathrm{Si}-\mathrm{O}$ set in motion by the conformational transitions of the preceding ( $\mathrm{Si}-\mathrm{O}, \mathrm{O}-\mathrm{Si})$ repeat unit. The solid and dashed curves are obtained with the respective sets 1 and 2 of energy parameters. A stretched exponential decay with power equal to 0.83 is found from the slope of the $\log [-\ln$ $M(t)]$ vs $\log t$ plot shown in the inset.

between the most distant microstates in a given ridge, is indicative of a random diffusion process, while passage between the closest microstates is governed by a linear proportionality between time and distance.

The time decay of the orientational autocorrelation function $M(t)$ for the bond vector $\mathrm{Si}-\mathrm{O}$ set in motion by the conformational transitions of the preceding ( $\mathrm{Si}-\mathrm{O}, \mathrm{O}-$ $\mathrm{Si}$ ) repeat unit is shown in Figure 11. The solid and dashed curves obtained with the respective sets 1 and 2 of energy parameters are very close to each other, although the frequency distribution of relaxational modes is different in the two cases. The fast transitions within a given ridge constitute the major process responsible for the loss of orientational correlation, and the full decay of $M(t)$ occurs before the slower mechanism of relaxation corresponding to passages between ridges can contribute effectively. The $\log [-\ln M(t)]$ vs $\log t$ plot shown in the inset in Figure 11 indicates the occurrence of a stretched exponential decay of orientational correlation, with power equal to 0.83 , as calculated from the slope of the best fitting straight line. A deviation from single-exponential behavior is common to all cooperative relaxation processes; here the exponent approaches unity since a relatively narrow distribution of relaxational modes, corresponding to transitions within a given ridge only, is predominantly operating.

\section{Conclusion}

In the present work, we focused on the conformational dynamics of disubstituted polysiloxanes, and PDtBS in particular, as an example of a chain which enjoys, on the one hand, a high degree of flexibility from the point of view of backbone bonds but is, on the other hand, constrained to perform highly coupled motions due to the steric interactions of the bulky side groups. Molecular dynamics simulations reveal two distinct types of coupling between near-neighbor bond rotations: First, the pairs of bonds about the oxygen atoms are found to undergo fast counterrotations of large amplitude, as illustrated in Figure $5 a, b$. These fast motions constitute the basic mechanism of the time decay of bond orientational autocorrelations according to dynamic rotational isomeric state considerations. Second, there is a totally different level of coupling between neighboring units, which occurs at time scales about 3 orders of magnitude slower than the first type of motions. This slower process of relaxation is identified with the passages between the high-probability ridges of the energy contour plots, which are constructed as a function of the two torsional angles of a pair of bonds centered about 0 . The fact that two adjacent repeat units undergo simultaneous passages between ridges in concerted fashion brings into consideration the strong coupling between bonds $i$ and $i+3$.

Acknowledgment. This research was supported by National Science Foundation Grant DMR 89-15025 and Department of Defense University Research Initiative 28747-MS-SM. Partial support from Bogazici University Research Grant 91P0028 is gratefully acknowledged by I.B.

\section{References and Notes}

(1) Neuburger, N.; Bahar, I.; Mattice, W. L. Macromolecules 1992, $25,2447$.

(2) Cerf, R. Advances in ChemicalPhysics; Interscience: New York, 1975; Vol. XXXIII, p 73

(3) Computer Simulation of Polymers; Roe, R. J., Ed.; PrenticeHall: Englewood Cliffs, NJ, 1991.

(4) Dodge, R.; Mattice, W. L. Macromolecules 1991, 24, 2709.

(5) Zhan, Y.; Mattice, W. L. Macromolecules 1992, 25, 1554.

(6) Flory, P. J. Statistical Mechanics of Chain Molecules; Interscience: New York, 1969.

(7) Glauber, R. J. J. Math Phys. 1963, 4, 294. Skinner, J. L. J. Chem. Phys. 1983, 79, 1955.

(8) Jernigan, R. L. In Dielectric Properties of Polymers; Karasz, F. E., Ed.; Plenum: New York, 1972; p 99.

(9) Bahar, I.; Erman, B. Macromolecules 1987, 20, 1368.

(10) Helfand, E. Science 1984, 226, 647.

(11) Doherty, D. C.; Hopfinger, A. J. Macromolecules 1990, $23,676$.

(12) Zuniga, I.; Bahar, I.; Dodge, R.; Mattice, W. L. J. Chem. Phys. $1991,95,5348$.

(13) Valeur, B.; Jarry, J. P.; Gény, F.; Monnerie, L. J. Polym. Sci., Polym. Phys. Ed. 1975, 13,667.

(14) Boyer, R. F. Rubber Chem. Technol. 1963, 34, 1303. Schatzki, T. F. J. Polym. Sci. 1962, 57, 496.

(15) Those intervals follow from the scheme depicted in Figure 3. It is noted from Figure $4 \mathrm{~b}$, however, that, throughout the MD simulations, the diagonal projection of ridge $\mathrm{A}^{\prime}$ on the righthand coordinate remains in the more restricted range of $90^{\circ} \leq$ $\Psi \leq 130^{\circ}$, approximately, inasmuch as larger $\Psi$ values are associated with very high energy states.

(16) The solid $C(4, t / 1,0)$ curve exhibits a maximum at $t=10.8 \mathrm{ps}$, which is hardly discernible in the graph. The corresponding dashed curve is monotonically strictly increasing. 